\title{
INTEGRATING ADDITIONAL KNOWLEDGE INTO ESTIMATION OF GRAPHICAL MODELS
}

\author{
By Yunqi Bu And Johannes Lederer \\ University of Washington
}

\begin{abstract}
In applications of graphical models, we typically have more information than just the samples themselves. A prime example is the estimation of brain connectivity networks based on fMRI data, where in addition to the samples themselves, the spatial positions of the measurements are readily available. With particular regard for this application, we are thus interested in ways to incorporate additional knowledge most effectively into graph estimation. Our approach to this is to make neighborhood selection receptive to additional knowledge by strengthening the role of the tuning parameters. We demonstrate that this concept (i) can improve reproducibility, (ii) is computationally convenient and efficient, and (iii) carries a lucid Bayesian interpretation. We specifically show that the approach provides effective estimations of brain connectivity graphs from fMRI data. However, providing a general scheme for the inclusion of additional knowledge, our concept is expected to have applications in a wide range of domains.
\end{abstract}

1. Introduction. Brain connectivity networks derived from fMRI data are considered a prime gateway to understanding cognitive diseases. Along with other fields, brain research has thus boosted the interest in statistical methodology for uncovering dependence networks. A standard framework for dependence networks is Gaussian graphical models [19]. Gaussian graphical models have become particularly popular after the development of methods and algorithms that can handle large and high-dimensional data.

Two widely-used approaches to Gaussian graphical models are neighborhood selection and graphical lasso. Neighborhood selection aims at graph reconstruction by aggregating local estimates [21]. Graphical lasso, on the other hand, is based on a global objective function [2, 9, 29]. Both approaches are now accompanied by a bulk of literature on theory and computation; we refer to $[5,15]$ and references therein. However, standard estimators do not take into account additional knowledge that is often available in practice, such as scientific rationales, experimental arrangements, or insights from

MSC 2010 subject classifications: Primary 62H20; secondary 62P10

Keywords and phrases: brain connectivity networks, reproducible graph estimation, additional knowledge in tuning parameters 
previous studies. A natural question is thus how graph estimation can be refined when additional knowledge is available.

The purpose of this paper is to study this question. Our main inspiration and application are brain connectivity networks. In particular, we are interested in estimating brain connectivity networks by analyzing resting-state functional magnetic resonance imaging (fMRI) data that describe the levels of co-activation between brain regions [28] as measured by changes in blood flow [17]. Brain regions have spatial coordinates, so in addition to the samples, there is information in terms of pairwise distances between regions. Our goal is to leverage this additional knowledge for effective graph estimation.

Our main idea for this is to strengthen the role of tuning parameters. Commonly, tuning parameters are considered an inconvenience, because they need to be calibrated for each data set specifically. We instead think of this adaptability as an asset that can make tuning parameters a potent instrument for funneling external information into the estimation process. More specifically, for our goal of brain network estimation, we use tuning parameters to make neighborhood selection receptive to additional knowledge. We first show numerically that without such an integration, the sample sizes needed for accurate graph recovery are surprisingly large. We then show that adopting our notion can lead to three main features.

Reproducibility: Reproducibility is a major principle in the sciences and has become again a heated topic in recent literature [7, 23, 25]. We thus adopt a notion of reproducibility for our data application to show the competitiveness of our approach.

Implementation: We point out that our approach is amenable to straightforward and efficient implementations based on existing software. In particular, our approach preserves the general forms of the standard penalties and does not introduce any additional penalty terms.

Interpretation: We demonstrate that our approach has a lucid Bayesian interpretation.

The remainder of the paper is structured as follows. In Section 2, we introduce and motivate our general concept. In Section 3, we then tailor this concept to the estimation of brain connectivity networks based on fMRI data. In Section 4, we conclude with a discussion.

2. Method. We start with a brief review of Gaussian graphical models. We then demonstrate the need for additional knowledge in graph estimation with a simulation study that adopts the dimensions of the brain connectivity application. We are then ready to introduce and discuss our approach for amending the estimation process with additional knowledge. We finally con- 
firm by using a simulation that our method can improve graph estimation.

2.1. A brief review of Gaussian graphical models. Gaussian graphical models assume samples from a centered $p$-dimensional normal distribution $\mathcal{N}_{p}(0, \Sigma)$ with a symmetric, positive definite covariance matrix $\Sigma$. The distribution is then complemented with an associated undirected graph $\mathcal{G}=(\mathcal{V}, \mathcal{E})$ that has node set $\mathcal{V}=\{1, \ldots, p\}$ and edge set $\mathcal{E}=\left\{(i, j) \in \mathcal{V} \times \mathcal{V}:\left(\Sigma^{-1}\right)_{i j} \neq\right.$ $0\}$.

The crux of Gaussian graphical models is that the conditional and marginal dependence structures of the samples are concisely captured by the edge set $\mathcal{E}$. Indeed, the Hammersley-Clifford theorem $[4,13]$ states that the $i$ th and $j$ th coordinate of a sample from $\mathcal{N}_{p}(0, \Sigma)$ are conditionally independent given all other coordinates if and only if $\left(\Sigma^{-1}\right)_{i j}=0$, that is, $(i, j) \notin \mathcal{E}$. Moreover, the $i$ th and $j$ th coordinate are independent if and only if one cannot construct a chain of the form $\left(i, k_{1}\right),\left(k_{1}, k_{2}\right), \ldots,\left(k_{l}, j\right)$ by using only elements in $\mathcal{E}$. Our goal is consequently to uncover the edge set $\mathcal{E}$ from data. For this aim, we develop estimators $\widehat{\mathcal{E}} \equiv \widehat{\mathcal{E}}(X)$ of $\mathcal{E}$ based on independent identically distributed Gaussian samples $X_{1}, \ldots, X_{n} \in \mathbb{R}^{p}$ summarized in the data matrix $X=\left(X_{1}, \ldots, X_{n}\right)^{\top} \in \mathbb{R}^{n \times p}$.

2.2. The need for additional knowledge. We now demonstrate that even with optimal tuning and large sample sizes, standard methods for Gaussian graphical modeling can fail to provide accurate graph recovery. For this, we conduct a simulation study comparing four of the most popular methods: thresholding the partial correlation matrix (THR); neighborhood selection with the "or-rule" (MB(or)); neighborhood selection with the "and-rule" (MB(and)); and graphical lasso (GLASSO).

We simulate data with the aim of mimicking settings encountered in practice. For this, the number of nodes is set to $p=116$, which equals the number of brain regions in the data considered in Section 3. Then, a standard preferential attachment algorithm [3] is used to construct 115 edges between these nodes. The resulting edge set $\mathcal{E}$ determines which off-diagonal entries of the inverse covariance matrix $\Sigma^{-1}$ are non-zero. The values of these entries are then independently sampled uniformly at random from $[-1,-0.2] \cup[0.2,1]$. Diagonal entries of $\Sigma^{-1}$ are set to a common value such that the condition number equals 100 . With $\Sigma^{-1}$ constructed this way, vectors are independently sampled from the Gaussian distribution $\mathcal{N}_{p}(0, \Sigma)$ and summarized in data sets with sample sizes $n \in\{50,100,200,400,600,800,1000,1200$, $1400,1600\}$.

The accuracy of graph recovery is assessed via Hamming distance. The Hamming distance between the estimated edge sets $\tilde{\mathcal{E}}$ and the true edge 
set $\mathcal{E}$ is defined by $d_{\mathrm{H}}(\tilde{\mathcal{E}}, \mathcal{E}):=\mid\{(i, j):(i, j) \in \tilde{\mathcal{E}},(i, j) \notin \mathcal{E}\} \cup\{(i, j):(i, j) \notin$ $\tilde{\mathcal{E}},(i, j) \in \mathcal{E}\} \mid$. Larger Hamming distances indicate less accurate estimation. The tuning parameters of all methods are calibrated to minimal Hamming distance, noting that the true graphs are known in simulations. This "oracle" tuning allows us to study the maximal potential of the methods' accuracies.

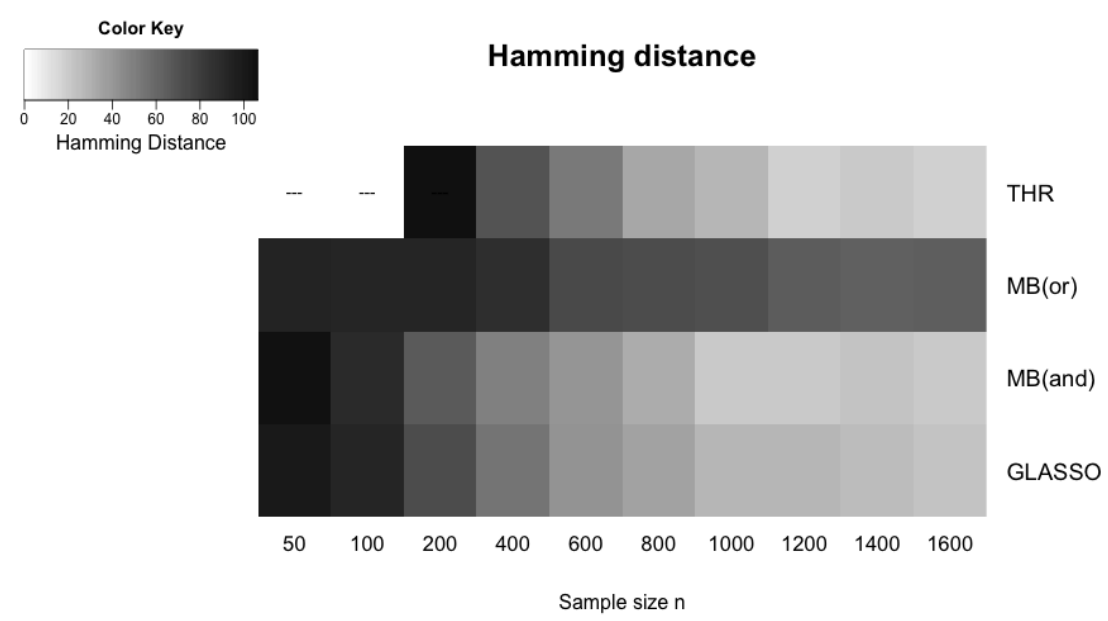

Fig 1: Graph estimation with optimally calibrated standard methods becomes more accurate as the sample size $n$ increases. However, even with $n=1600$, which is much larger than the number of covariates $p=116$, the Hamming distances are 32 or above. This means that graph estimation with standard methods can still be highly inaccurate even when the sample sizes are very large.

Figure 1 contains the heat-map for accuracies averaged over 20 repetitions. For $n=1600$, THR is the most accurate approach among all four methods, having average Hamming distance 32. Observe, however, that 32 wrongly assigned edges still mark a poor performance given that there are only 115 true edges in total. For smaller sample sizes, the accuracies of the methods decline even further (note that in particular, THR requires $p \leq n$ and thus can not be applied in the $n \in\{50,100\}$ regime as indicated by dashed lines in the figure). In view of real data being commonly high-dimensional, where $p$ is of the same order as $n$ - or even larger, these observations thus provide substantial motivation for the inclusion of additional knowledge.

2.3. Neighborhood selection with additional knowledge. We now introduce our scheme to incorporate additional knowledge into graph recovery. The resulting method can be computed efficiently with standard software 
packages and has a direct Bayesian interpretation.

A basis particularly suited for our approach is neighborhood selection. To recapitulate, the main idea of neighborhood selection is that graph recovery can be established through a sequence of regressions. The corresponding regression parameter $\beta^{j} \in \mathbb{R}^{p}$ for a given node $j$ determines the edges between node $j$ and the other nodes: $\left(\beta^{j}\right)_{i} \neq 0 \Leftrightarrow(i, j) \in \mathcal{E}$. With this in mind, the standard estimators are of the form

$$
\hat{\beta}^{j, r^{j}} \in \underset{\substack{\beta \in \mathbb{R}^{p} \\ \beta_{j}=0}}{\operatorname{argmin}}\left\{\frac{1}{2}\left\|X^{j}-X \beta\right\|_{2}^{2}+r^{j}\|\beta\|\right\}, \quad(j \in\{1, \ldots, p\}) .
$$

Here, the vector $X^{j} \in \mathbb{R}^{n}$ denotes the $j$ th column of the data matrix $X$, the positive numbers $r^{1}, \ldots, r^{p} \in[0, \infty)$ are tuning parameters, and $\|\cdot\|$ is a norm (or more generally, a convex, positive function, such as the Ridge penalty). A standard example is neighborhood selection with the lasso, where $\|\cdot\|$ is set to the $\ell_{1}$-norm. Adopting the "and-rule", the edge set $\mathcal{E}$ is finally estimated by $\hat{\mathcal{E}}=\left\{(i, j) \mid\left(\hat{\beta}^{i, r^{i}}\right)_{j} \neq 0\right.$ and $\left.\left(\hat{\beta}^{j, r^{j}}\right)_{i} \neq 0\right\}$.

The choice of $\mathrm{MB}$ (and) as the basis is motivated by the simulation results in Section 2.2 and the fact that there is a myriad of existing software packages for solving problems of the form (2.1). In principle, however, one could apply the following recipe also to $\mathrm{MB}$ (or) and GLASSO.

Our proposal is now to incorporate additional knowledge by "upgrading" the univariate tuning parameters $r^{1}, \ldots, r^{p}$ to vectors. For this, we assume additional knowledge in the form of a matrix $D \in \mathbb{R}^{p \times p}$. For example, $D_{i j}$ could be the Euclidean distance between nodes $i$ and $j$ in cases where the nodes correspond to brain regions, countries, galaxies, etc. As another example, $D_{i j}$ could be the (conditional) correlation between nodes $i$ and $j$ estimated on the present data set with an initial estimator (specializing our method to adaptive lasso-type approaches [30], for example) or more interestingly, with the same or different estimators on other data sources. Next, to incorporate $D$ into neighborhood selection, we transform the onedimensional tuning parameters $r^{j} \in[0, \infty)$ into multi-dimensional tuning parameters $\mathbf{r}^{j} \in[0, \infty)^{p}$. We then enrich these tuning parameters with additional knowledge by setting $\left(\mathbf{r}^{j}\right)_{i}=\bar{r}^{j} \cdot f\left(D_{i j}\right)$, where $f: \mathbb{R} \rightarrow \mathbb{R}$ is a link function and $\bar{r}^{j}$ is an overall tuning parameter for the $j$ th regression. As customary in high-dimensional statistics, the free parameter $\bar{r}^{j}$ balances the weights of the data ( $X$ in our case) and the structural assumptions (captured by $\|\cdot\|$ and $D$ in our case). The above node-wise regression (2.1) is 
then generalized to

$$
\hat{\beta}^{j, \mathbf{r}^{j}} \in \underset{\substack{\beta \in \mathbb{R}^{p} \\ \beta_{j}=0}}{\operatorname{argmin}}\left\{\frac{1}{2}\left\|X^{j}-X \beta\right\|_{2}^{2}+\left\|\mathbf{r}^{j} \circ \beta\right\|\right\}, \quad(j \in\{1, \ldots, p\}),
$$

where the circle $\circ$ indicates element-wise multiplication.

Let us discuss three practical and methodological aspects of (2.2). The first important observation is that while there are now $p$ "tuning" parameters per regression, there is still only one free parameter (namely $\bar{r}^{j}$ ) that requires calibration. This is highly desirable: for one parameter, efficient calibration schemes are known [1, 6, 24]; in contrast, the calibration of multiple free parameters, which would appear when adding additional penalty terms instead of adopting our approach, remains a major challenge in both theory and computations. Second, for the link function $f$, there are often natural choices; we refer to the application section and the discussion. Third, note that our approach retains the "flavor" of the original penalty. This means that our concept maintains the general properties of the penalty, such as the sparsity generating effect of $\ell_{1}$-penalties [26], and it means that the practical implementation of (2.2) can be based on standard software packages, such as glmnet [10] in the case of $\ell_{1}$-penalization.

Finally, our approach has a direct Bayesian interpretation. For a given index $j$, we consider the hierarchical Bayesian model

$$
\begin{gathered}
X^{j} \mid \beta, \sigma \sim \mathcal{N}\left(X \beta, \sigma^{2} \mathrm{I}_{n \times n}\right), \\
\operatorname{Pr}\left(\beta_{i} \mid \sigma,\left(\mathbf{r}^{j}\right)_{i}\right)=\frac{\left(\mathbf{r}^{j}\right)_{i}}{2 \sigma^{2}} e^{-\frac{\left(\mathbf{r}^{j}\right)_{i}}{\sigma^{2}}\left|\beta_{i}\right|}, \quad(i \in\{1, \ldots, p\}) .
\end{gathered}
$$

This model is a generalization of the model considered in the seminal paper on the Bayesian lasso [22]. The parameters $\left(\mathbf{r}^{j}\right)_{1}, \ldots,\left(\mathbf{r}^{j}\right)_{p}$ in our general model are hyperparameters that specify the shape of the prior distribution of each of the regression coefficients $\left(\beta^{j}\right)_{i}$. The negative log-posterior distribution of $\beta$ is now given by

$$
-\log \mathbb{P}\left(\beta \mid X^{j}, \sigma, \mathbf{r}^{j}\right)=\frac{1}{\sigma^{2}}\left(\frac{1}{2}\left\|X^{j}-X \beta\right\|_{2}^{2}+\sum_{i=1}^{p}\left(\mathbf{r}^{j}\right)_{i}\left|\beta_{i}\right|\right)+c,
$$

where $c$ is a term independent of $\beta$. The mode of this distribution is

$$
\hat{\beta}^{j, \mathbf{r}^{j}} \in \underset{\substack{\beta \in \mathbb{R}^{p} \\ \beta_{j}=0}}{\operatorname{argmin}}\left\{\frac{1}{2}\left\|X^{j}-X \beta\right\|_{2}^{2}+\sum_{i=1}^{p}\left(\mathbf{r}^{j}\right)_{i}\left|\beta_{i}\right|\right\}, \quad(j \in\{1, \ldots, p\}),
$$


which equals the estimator yielded by our approach when the penalty in (2.2) is set to the $\ell_{1}$-norm. Similarly, replacing the double-exponential distribution in (2.4) with a Gaussian distribution, the posterior mode equals the estimators yielded by our approach when the penalty in (2.2) is set to the $\ell_{2}$-norm.

This analysis shows that the tuning parameters relate our frequentist and Bayesian notions about the additional knowledge. In our frequentist estimator, the larger $\left(\mathbf{r}^{j}\right)_{i}$, the more likely the edge $(i, j)$ is excluded from the estimate. In our Bayesian view, the larger $\left(\mathbf{r}^{j}\right)_{i}$, the more the assumed distribution of $\left(\beta^{j}\right)_{i}$ is concentrated around zero. Thus, funneling the additional knowledge into the tuning parameters of the frequentist estimator can be viewed as transforming the original priors, which are the same all across the coefficient vector, into informed priors tailored to each coordinate.

2.4. Simulation. We now confirm by using a simulation that our approach can harvest additional knowledge to improve graph estimation. To generate data, we imitate the brain connectivity application in Section 3: the number of nodes $p=116$ corresponds to the number of brain regions; the number of samples $n=210$ corresponds to the number of fMRI scans per subject; the additional knowledge $D_{i j}$ is the Euclidian distance between brain regions $i$ and $j$. The edge set is constructed based on independent $\operatorname{Bernoulli}\left(p_{i j}\right)$ distributions, $p_{i j}=$ inv.logit $\left(10-D_{i j} / 3\right)$, such that an edge $(i, j)$ is included if the corresponding Bernoulli outcome is one. The form of the distributions captures our rationale that direct connections are predominately between close regions. An anatomical map of a graph generated by the preceding scheme is displayed in the left panel of Figure 2. Next, the non-zero entries in the inverse covariance matrix $\Sigma^{-1}$ as specified by the edge set are set to 0.3 . The diagonal entries are set to $0.2+0.3 \cdot \sigma_{\min }$, where $\sigma_{\min }$ is the minimal singular value of the adjacency matrix. This construction ensures that $\Sigma^{-1}$ has full rank. Finally, $n$ i.i.d. samples are generated from $\mathcal{N}_{p}(0, \Sigma)$.

We now run our approach against standard methods for graph estimation. The specification of our estimator, referred to as SI in the following, is the same as in the application section, namely neighborhood selection based on (2.2) with $\ell_{1}$-norm penalty and link function $f(x)=x^{3}$; see Section 3.2 for further comments. The standard methods competing are THR, MB, and GLASSO. Motivated by the simulation results in Section 2.2, we adopt the "and-rule" for SI and MB. A total of 20 data sets is generated, on which each method is run as a function of its free tuning parameter. The average ROC curves for graph recovery are plotted in the right panel of Figure 2. We 

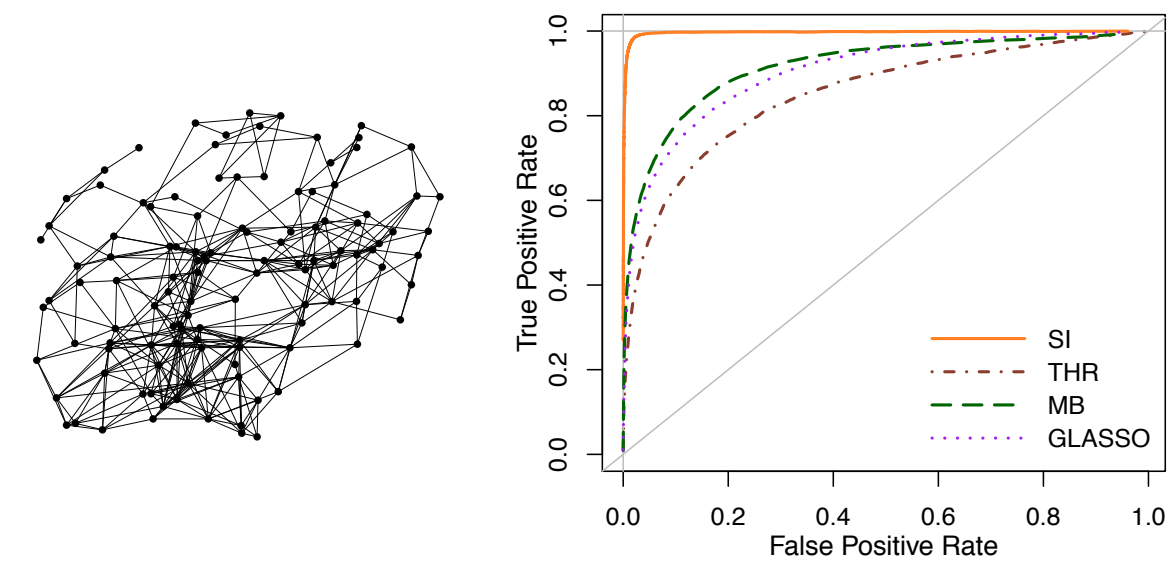

Fig 2: Left panel: One example of the 20 simulated graphs. Right panel: ROC curves demonstrating that SI can outperform standard methods when additional knowledge is available.

find that the ROC curve of SI dominates the other curves, demonstrating that SI can improve graph recovery when additional knowledge is available.

We finally note that simulations alone cannot provide definite evidence in favor of a method, because data generating processes always reflect preconceived notions about the biological truth. We thus complement the simulation with a reproducibility study on real data in the following section. Together, the results provide us with sufficient confidence to argue in favor of SI.

3. Brain connectivity application. The main motivation for the general framework in Section 2 is brain connectivity networks. Our biological rationale for these networks is that direct connections are more likely between close regions than between distant regions. The novel concept we proposed is ideal for incorporating this notion. First, graphical models in general distinguish between direct connections (conditional dependence) and indirect connections (marginal dependence). Second, the mentioned rationale that the distance between two nodes influences the likelihood of them being directly connected can be reflected naturally by how the link function $f$ incorporates the additional distance information $D$. Importantly, we do not generally exclude the direct or indirect long-range connections. This means that two distant nodes can very well be connected, but more likely, this connection will be indirect. While we work with specific data about Alzheimer's disease in the following, our main goal is to show that our method provides 
a sensible approach to brain connectivity in general.

3.1. Background and data set. Functional Magnetic Resonance Imaging (fMRI) is a promising gateway to the understanding of the human brain [28]. Our specific goal is to use brain activity records from fMRI to infer coactivation networks among brain regions. For this, we rely on data collected from outpatients at the Neurology Department of the Beijing Hospital from November 2013 through December 2015. The data set comprises 37 subjects: 22 patients with Alzheimer's disease (AD), 5 patients with mild cognitive impairment (MCI), and 10 patients with normal cognition (NC). Each subject's data contains $n=210$ consecutive scans of the entire brain. Each of the $p=116$ variables is an average intensity over all voxels in an anatomical volume of interest defined by Automated Anatomical Labeling [27]. An autoregressive integrated moving average model $[12,16]$ is applied to account for autocorrelation.

3.2. Specification of the method. We now reconcile the general methodology with the biological rationale of the application. For this, we consider the estimators $(2.2)$ with $\ell_{1}$-penalization to exploit potential sparsity of the graphs. The additional knowledge $D_{i j}$ is the Euclidian distance between brain regions $i, j$, and the link function is defined as $f(x)=x^{3}$ to capture the three-dimensionality of the coordinates. This yields tuning parameters of the form $\left(\mathbf{r}^{j}\right)_{i}=\bar{r}^{j} \cdot D_{i j}^{3}$. We compare our approach SI to THR, MB, and GLASSO. Again, we adopt the "and-rule" for SI and MB. The tuning parameter in MB as well as $\bar{r}^{j}$ in SI are selected via 10-fold cross-validation. Since the tuning parameter in GLASSO is not amenable to the same crossvalidation scheme, GLASSO is calibrated via BIC. In absence of established selection criteria for thresholding, THR is calibrated such that the number of connections equals the number of connections for SI.

Note that the coordinates of the observations in the present data set correspond to volume regions in the brain. The observations in another type of fMRI-induced data that received considerable attention recently correspond to the cerebral cortex [11]. We would then recommend our approach with geodesic distances among the regions as additional knowledge and a link function of the form $f(x)=x^{2}$ to capture the two-dimensionality of the spherical coordinates.

3.3. Reproducibility study. Reproducibility is a basic scientific principle. It requires that experiments and analyses can be replicated, and that these replications lead to the same scientific conclusions. This means in particular that the statistical methods involved need to be stable in the sense that 
TABLE 1

SI has higher reproducibility on the fMRI data set than standard methods

\begin{tabular}{lc}
\hline Method & Reproducibility (SD) \\
\hline SI & $62(7)$ \\
MB & $42(6)$ \\
GLASSO & $55(7)$ \\
\hline
\end{tabular}

two estimates should be commensurate if the corresponding data sets are collected in the same fashion.

An effective way to probe reproducibility of a method is data splitting. Indeed, agreement of estimates based on two parts of a data set under consideration indicates that the method is stable, while largely disagreeing estimates raise a red flag. To compare the reproducibility of different methods on the fMRI data, we proceed as follows. Given a patient and a method, the data describing the 210 samples are split randomly into two sets of 105 samples; then, reproducibility in terms of percentage agreement $\left(1-d_{\mathrm{H}}(\hat{\mathcal{E}}, \tilde{\mathcal{E}}) /(|\hat{\mathcal{E}}|+|\tilde{\mathcal{E}}|)\right) \%$ is calculated for the graphs $\hat{\mathcal{E}}$ and $\tilde{\mathcal{E}}$ that are estimated on the two halves of the data. Averages are finally taken over 20 random splits and over all the patients.

Table 1 shows that SI has the highest reproducibility among the method considered. (Note that the sample sizes in the split data are not sufficiently large for the inclusion of THR.) This suggests that the additional knowledge is integrated effectively and that SI makes graph estimation on the data set under consideration more reliable.

3.4. Connectivity remarks. After comparing the methods in terms of reproducibility, we give some brief remarks about the final graph estimates.

To highlight differences among the methods, graphs for one subject from each of the study groups are displayed in Figure 3. The graphs are estimated based on all the data available for one given subject at a time. Each column in the figure corresponds to one (fixed) subject of the three groups AD, MCI, and NC; each row corresponds to one of the four methods SI, THR, MB, and GLASSO. The plots show anatomical maps, that is, the nodes are positioned according to their 3D coordinates. Edges between near regions (distances in the lower quartile) are colored orange; edges between more remote regions are colored blue. The total number of edges is stated below each map. While showing only one set of graphs for illustration, we find two patterns across all subjects: (i) With cross-validation as the most common calibration scheme applied, SI yields the most sparse networks. This finding shows that SI can lead to more manageable models. (ii) SI yields a small number of edges 
between distant regions, but most edges are between spatially close regions. In strong contrast, the other methods do not exhibit such a preference. This finding confirms our expectations about the four methods, and it shows that the estimates provided by SI are in agreement with the biological rationale.

To highlight differences among the study groups, the differences between the average graph estimates of SI for the AD and NC groups are displayed in Figure 4. Since accurate graph estimation is the basis for accurate group comparisons, we expect that SI can help uncovering differences and similarities among study groups in general. In the following, we briefly discuss some insights obtained for the data set at hand, leaving a complete biological study for future work. Note first that entries in the heat-map in Figure 4 denote the frequencies of the edges in the $\mathrm{AD}$ group minus the corresponding frequencies in the NC group. The columns and rows are arranged such that spatially close regions tend to be close in the figure. We make two observations: (i) The overall graphs for the two groups seem to be similar, but there also seem to be pronounced differences in the connectivities of some regions. This finding suggests that some connections might be affected by AD more than others. (ii) Differences are found predominantly between spatially close regions (see blue and yellow entries close to the diagonal). This finding hints at the possibility that $\mathrm{AD}$ concerns short-range direct connections more than long-range direct connections. (iii) The plot contains areas with high variability (blocks with high concentrations of blue and yellow entries) such as the cerebelum close to the bottom right corner. The plot thus locates areas of interests for further studies.

4. Discussion and further research. We have shown that strengthening the role of tuning parameters is an effective approach to incorporating additional knowledge. In particular, we have shown that this approach can improve reproducibility, is computationally convenient, and has a clear Bayesian interpretation.

Our scheme allows for the inclusion of application-specific information matrices $D$ and link functions $f$. The form of the information matrix $D$ is typically predetermined in a given application. Similarly, the form of $f$ often follows clear scientific rationales as can be seen in our brain connectivity example. One could also consider data-driven selections of $f$ from a set of candidate functions by using score testing or related methods, cf. [14]; however, the sample sizes of typical data might be too small for a thorough non-parametric selection of the link function. Importantly, via the link function $f$ and the information matrix $D$, our approach can be tailored to a wide range of application in bioinformatics, speech recognition, computer 
vision, and digital communications. For example, we envision further implementation in genomics, where the goal is to estimate gene regulatory networks based on gene expression levels [8]. The additional knowledge that researchers commonly want to invoke for this are previously established subnetworks or networks estimated in other studies.

Finally, another feature of our concept is that it is readily amenable to theory and further extensions. We expect that sharp theoretical guarantees can be proved using standard techniques in the field [5]. Extensions to heavytailed or otherwise non-Gaussian data could be approached based on [20]. For the development of confidence sets for graph estimation with additional knowledge, we expect to combine our ideas with [18].

\section{ACKNOWLEDGEMENTS}

We thank Andrew Zhou for the inspiring discussions and Rosemary Adams for contributions to the visualizations. We also thank Noah Simon, Sam Koelle, and Mengjie Pan for helpful suggestions.

\section{REFERENCES}

[1] Arlot, S. and Celisse, A. (2010). A survey of cross-validation procedures for model selection. Statistics Surveys 4 40-79. MR2602303

[2] Banerjee, O., Ghaoui, L. E. and D'Aspremont, A. (2008). Model selection through sparse maximum likelihood estimation for multivariate Gaussian or binary data. The Journal of Machine Learning Research 9 485-516. MR2417243

[3] Barabási, A. L. and Albert, R. (1999). Emergence of scaling in random networks. Science 286 (5439), 509-512. MR2091634

[4] Besag, J. (1974). Spatial interaction and the statistical analysis of lattice systems. Journal of the Royal Statistical Society, Series B (Methodological) 36 192-236. MR0373208

[5] Bühlmann, P. and van de Geer, S. (2011). Statistics for High-Dimensional Data: Methods, Theory and Applications. Springer Science and Business Media, Heidelberg Dordrecht London New York. MR2807761

[6] Chichignoud, M., Lederer, J. and Wainwright, M. J. (2016). A practical scheme and fast algorithm to tune the lasso with optimality guarantees. Journal of Machine Learning Research. 17(231), 1-20.

[7] Collins, F. S. and TABAK, L. A. (2014). NIH plans to enhance reproducibility. Nature $\mathbf{5 0 5}(7485), 612$.

[8] Filkov, V. (2005). Identifying gene regulatory networks from gene expression data. Handbook of Computational Molecular Biology 27-1. Chapman and Hall/CRC. MR2229200

[9] Friedman, J., Hastie, T. and Tibshirani, R. (2008). Sparse inverse covariance estimation with the graphical lasso. Biostatistics 9(3), 432-441.

[10] Friedman, J., Hastie, T. and Tibshirani, R. (2010). Regularization paths for generalized linear models via coordinate descent. Journal of Statistical Software 33(1), 1-22. http://www.jstatsoft.org/v33/i01/ 
[11] Glasser, M. F., Coalson, T. S., Robinson, E. C., Hacker, C. D., Harwell, J., Yacoub, E., Ugurbil, K., Andersson, J., Beckmann, C. F., Jenkinson, M. and Smith, S. M. (2016). A multi-modal parcellation of human cerebral cortex. Nature. 536(1), 171-178.

[12] Granger, C. W. and Morris, M. J. (1976). Time series modeling and interpretation. Journal of the Royal Statistical Society, Series A (General) 246-257. MR0461816

[13] Grimmett, G. R. (1973). A theorem about random fields. Bulletin of the London Mathematical Society 5(1), 81-84. MR0329039

[14] Gu, Q., CaO, Y., Ning, Y. and LiU, H. (2015). Local and global inference for high dimensional gaussian copula graphical models. arXiv:1502.02347.

[15] Hastie, T., Tibshirani, R. and Wainwright, M. (2015). Statistical Learning with Sparsity. CRC press, FL.

[16] Haugh, L. D. (1976). Checking the independence of two covariance-stationary time series: a univariate residual cross-correlation approach. Journal of the American Statistical Association 71(354), 378-385. MR0418379

[17] Huettel, S. A., Song, A. W., McCarthy, G. (2009). Functional Magnetic Resonance Imaging, Vol. 1. 2nd ed. Sinauer Associates, Sunderland.

[18] Janková, J. and VAN DE GEeR, S. (2015). Confidence intervals for high-dimensional inverse covariance estimation. Electronic Journal of Statistics 9(1), 1205-1229. MR3354336

[19] Lauritzen, S. L. (1996). Graphical Models. Oxford University Press, Clarendon. MR1419991

[20] Lederer, J. (2016). Graphical models for discrete and continuous data. arXiv:1609.05551.

[21] Meinshausen, N. and Bühlmann, P. (2006). High-dimensional graphs and variable selection with the lasso. Annals of Statistics 1436-1462. MR2278363

[22] Park, T. and Casella, G. (2008). The Bayesian lasso. Journal of the American Statistical Association 103(482), 681-686. MR2524001

[23] Peng, R. D. and Casella, G. (2009). Reproducible research and biostatistics. Biostatistics 10(3), 405-408.

[24] Sabourin, J., Valdar, W. and Nobel, A. (2015). A permutation approach for selecting the penalty parameter in penalized model selection. Biometrics 71 11851194. MR3436744

[25] Stodden, V., Leisch, F. and Peng, R. D. (2014). Implementing reproducible research. Chapman and Hall/CRC.

[26] Tibshirani, R. (1996). Regression shrinkage and selection via the lasso. Journal of the Royal Statistical Society, Series B (Methodological) 58 267-288. MR1379242

[27] Tzourio-Mazoyer, N., Landeau, B., Papathanassiou, D., Crivello, F., Etard, O., Delcroix, N., Mazoyer, B. and Joliot, M. (2002). Automated anatomical labeling of activations in SPM using a macroscopic anatomical parcellation of the MNI MRI single-subject brain. Neuroimage 15(1), 273-289.

[28] van Den Heuvel, M. P. and POL, H. E. H. (2010). Exploring the brain network: a review on resting-state fMRI functional connectivity. European Neuropsychopharmacology 20(8), 519-534.

[29] YUAN, M. and Lin, Y. (2007). Model selection and estimation in the Gaussian graphical model. Biometrika 94(1), 19-35. MR2367824

[30] Zou, H. (2006). The adaptive lasso and its oracle properties. Journal of the American statistical association 101(476), 1418-1429. MR2279469 
YUNQI Bu

Department of Biostatistics

UNIVERSITY OF WASHINGTON

Seattle, WA 98195, U.S.A

E-MAIL: yunqibu@uw.edu

\author{
JOHANNES LEDERER \\ Department of Statistics, \\ Biostatistics \\ UNIVERSITY OF WASHINGTON \\ SeAtTle, WA 98195, U.S.A. \\ E-MAIL: ledererj@uw.edu \\ URL: http://www.johanneslederer.com
}



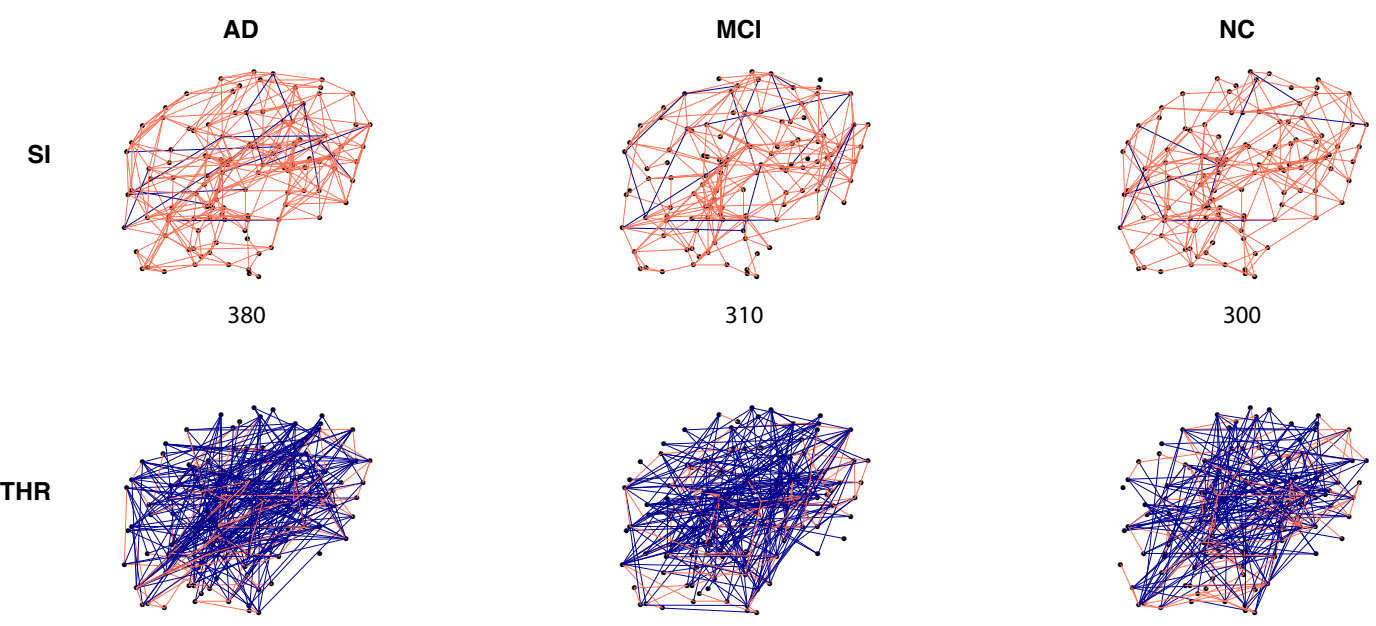

380

310

300

MB
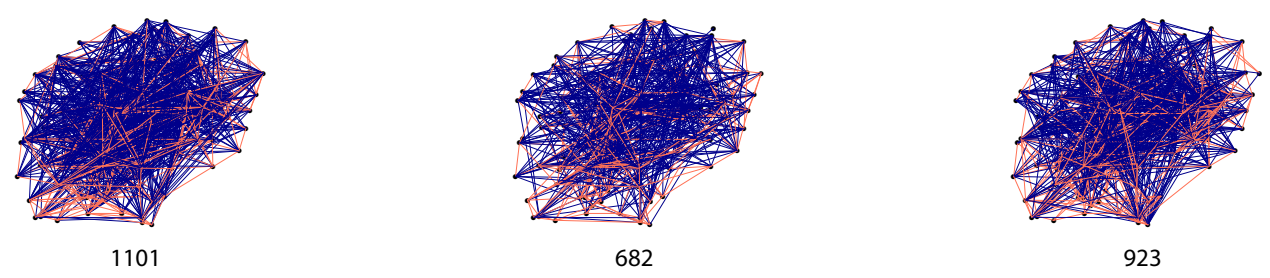

GLASSO
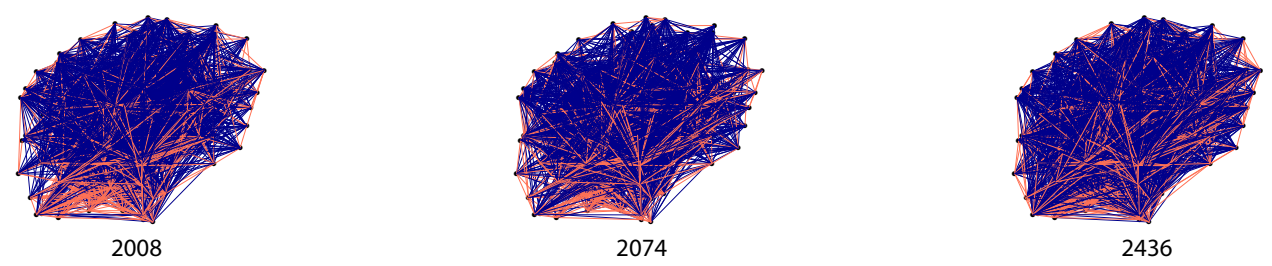

Fig 3: Anatomical maps of the estimated brain connectivity networks show that in contrast to standard methods, SI entails direct connections mostly between spatially close regions (orange lines). 


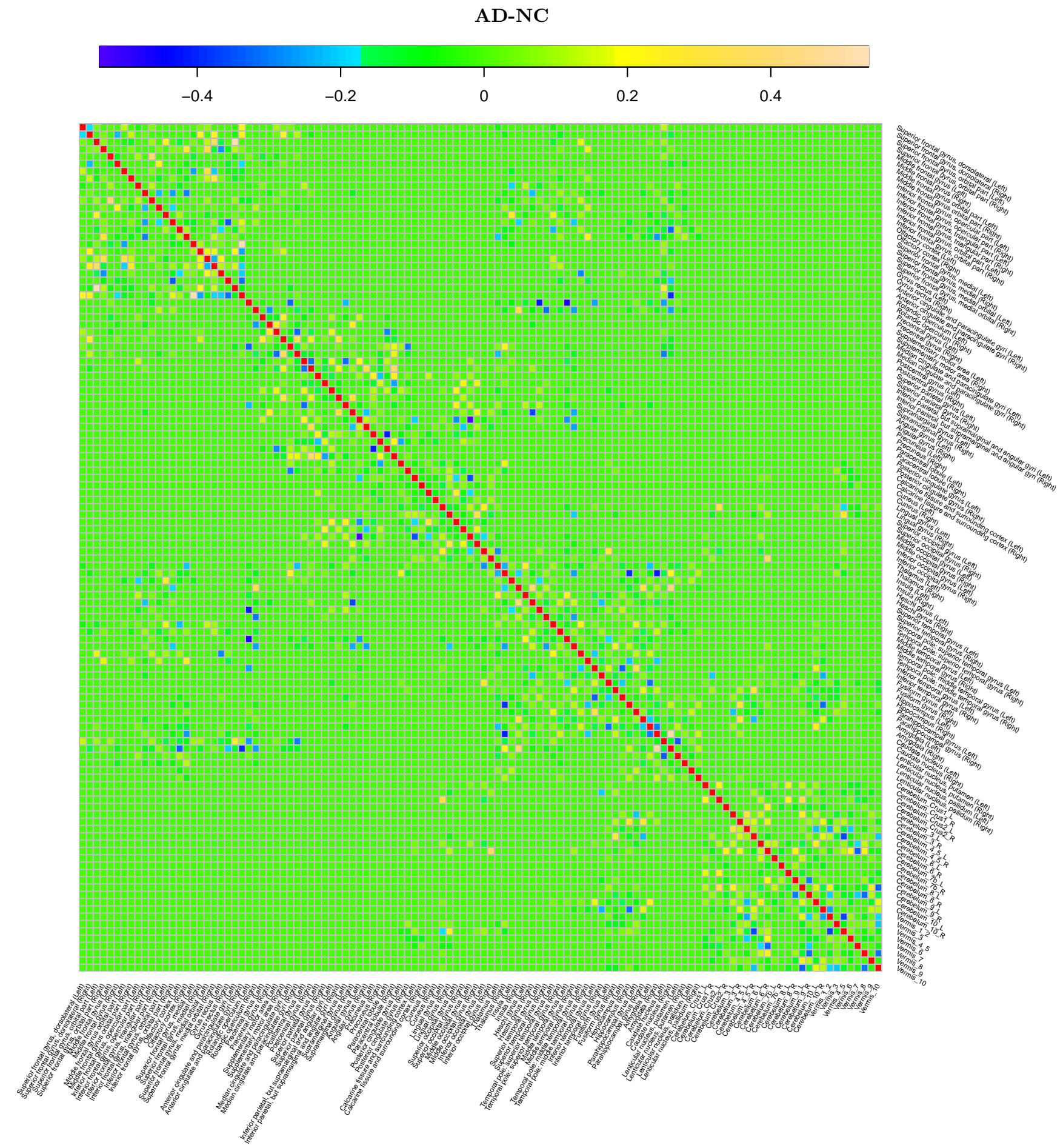

Fig 4: Differences of average brain connectivity networks between the AD group and the NC group. Yellow indicates that an edge occurs more frequently in the $\mathrm{AD}$ group; blue indicates that an edge occurs more frequently in the NC group. The diagonal is colored in red. While the networks seem similar overall, there also seem to be considerably different connectivities between some spatically close regions. 